\title{
Zur Problematik von Gewichtsangaben bei Rollmops
}

\author{
Dr. V. Etzel, M. Berges und S. Ramdohr \\ Niedersächsisches Landesamt für Verbraucherschutz und Lebensmittelsicherheit, Cuxhaven (LAVES)
}

\section{Zusammenfassung/Erläuterung}

Vor kurzem wurde die Frage der korrekten Deklaration des Fischanteils bei Rollmops aufgeworfen. Zwei Möglichkeiten wurden diskutiert. Entsprechend den Leitsätzen für Fische, Krebs- und Weichtiere und Erzeugnisse daraus (Fassung vom 27. Nov. 2002) wird ROLLMOPS

- nach II H 2a definiert als ausgenommener, entgräteter Hering ohne Kopf und Schwanzflosse, mit darin eingerollten Gewürzen und sonstigen pflanzlichen Beigaben [Die Füllung übersteigt nicht 20 Prozent des Rollmopsgewichtes. Die zum Durchspießen verwendeten Stäbchen bestehen aus gesundem, sauberem Holz oder aus Kunststoff.] und

- nach 3. das Fischgewicht das Gewicht des bei a) aus dem Fertigerzeugnis abgetropften, bei b) und c) eingewogenen Fisches oder Fischerzeugnisses beträgt, bezogen auf die Nennfüllmenge: a) bei Marinaden in Aufgüssen mindestens 50 Prozent.

Deklarationsmöglichkeiten:

1. Nach $\S 8$ der Lebensmittel-Kennzeichnungs-VO muss eine quantitative Zutatenkennzeichnung vorgenommen werden z. B. Verkehrsbezeichnung: Rollmops Zutaten: Hering (80\%), ausgenommen, entgrätet, Gurken, Essig, Salz, Gewürze
2. Eine Ausnahme von der Verpflichtung zur Mengenkennzeichnung besteht bei Zutaten oder Gattungen von Zutaten, deren Abtropfgewicht nach § 11 Abs. 1 FertigpackungsVO angegeben ist. Nach $\S 11$ Abs. 1 Fertigpackungs-VO ist bei festen Lebensmitteln in Aufgussflüssigkeiten neben der gesamten Füllmenge auch das Abtropfgewicht anzugeben (z. B. Füllgewicht: 1000 g; Abtropfgewicht: 600 g). Als Aufgussflüssigkeit zählen gemäß §11 Erzeugnisse, die gegenüber den wesentlichen Bestandteilen der betreffenden Zubereitung nur von untergeordneter Bedeutung und für den Kauf nicht ausschlaggebend sind.

Für das Fischkompetenzzentrum Nord ist der Hering als wesentliche Zutat anzusehen und entsprechend § 8 LMKV mengenmäßig zu kennzeichnen.

\section{Beschluss (einstimmig)}

Der Hering ist als wesentliche Zutat zum Erzeugnis Rollmops anzusehen und entsprechend §8 LMKV mengenmäßig zu kennzeichnen. 